\title{
Uninodular Fibromyxomatous Gastric Tumor Resected by Endoscopic Submucosal Dissection
}

\author{
Ikuhiro Kobori ${ }^{1}$, Yasumi Katayama ${ }^{1,2}$, Kazunori Hayashi ${ }^{1}$, Yo Fujimoto ${ }^{1}$, Mayuko Kaneko ${ }^{1}$, \\ Akihiro Kitahama ${ }^{1}$, Tomoyuki Kitagawa ${ }^{1}$, Hiroki Imada ${ }^{3}$, Shinichi Ban $^{3}$ and Masaya Tamano ${ }^{1}$
}

\begin{abstract}
:
Esophagogastroduodenoscopy of a 45-year-old woman revealed a submucosal tumor in the gastric antrum. Endoscopic submucosal dissection of the tumor was performed. The histological findings revealed a fibromyxomatous tumor composed of myofibroblastic cells with no evidence of malignancy. The growth pattern of the resected specimen was not multinodular or plexiform. We therefore tentatively referred to the present tumor descriptively as a gastric uninodular fibromyxomatous tumor, stressing its singular nodularity. It was initially roughly $10 \mathrm{~mm}$ in size but grew over a period of 4 years. A uninodular plexiform fibromyxoma might increase in size but might not become multinodular if it remains small.
\end{abstract}

Key words: mesenchymal mass, myofibroblastic cell, endoscopic submucosal dissection

(Intern Med 58: 2015-2018, 2019)

(DOI: 10.2169/internalmedicine.2370-18)

\section{Introduction}

Many gastric mesenchymal tumors are gastrointestinal stromal tumors (GISTs), and plexiform fibromyxoma is a very rare type with multiple nodular lesions $(1,2)$, the reported average size of which is $52 \mathrm{~mm}$ (3). This tumor is generally diagnosed and treated when it is relatively large. We herein report a uninodular gastric fibromyxoma with a diameter of $11 \mathrm{~mm}$ that was treated by endoscopic submucosal dissection (ESD).

\section{Case Report}

A 45-year-old woman visited a local physician with a chief complaint of pharyngeal discomfort. Esophagogastroduodenoscopy (EGD) revealed a submucosal tumor in the gastric antrum. The patient was referred to our hospital for a further workup. The findings of a physical examination and blood tests were normal, and she was negative for serum anti-Helicobacter pylori antibody. Repeat EGD revealed a submucosal tumor of approximately $10 \mathrm{~mm}$ in diameter with a slightly depressed center in the greater curvature of the gastric antrum (Fig. 1A), but image-enhanced endoscopy did not identify any mucosal irregularities (Fig. 1B). This patient had been examined at our institution four years earlier, and the tumor had obviously grown since then (Fig. 1C). The endoscopic ultrasonography (EUS) findings revealed a low-echoic mass that was continuous with the second layer of the gastric wall but without disruption of the third layer (Fig. 1D). Abdominal computed tomography (CT) revealed a tumor with a contrast effect in the gastric antrum and no obvious metastasis findings. A bite biopsy indicated a fibromuscular tumor, but we could not confirm this, and it was difficult to perform endoscopic ultrasound-guided fine needle aspiration (EUS-FNA) because of its small size.

Because there was a possibility that the tumor might grow larger and become malignant, we decided to excise the tumor. The patient gave her written informed consent to undergo ESD, which was performed without complications. Her postoperative course was good, and she was discharged on postoperative day six. The pathological findings revealed a dome-shaped polypoid lesion measuring $11 \times 11 \mathrm{~mm}$ with an apical ulcerative depression (Fig. 2A). The wellcircumscribed, uninodular tumor had a fibromyxomatous cut surface and was located mainly in the submucosa with mu-

${ }^{1}$ Department of Gastroenterology, Dokkyo Medical University Saitama Medical Center, Japan, ${ }^{2}$ Endoscopy Center, Dokkyo Medical University Saitama Medical Center, Japan and ${ }^{3}$ Department of Pathology, Dokkyo Medical University Saitama Medical Center, Japan

Received: November 11, 2018; Accepted: January 16, 2019; Advance Publication by J-STAGE: March 28, 2019

Correspondence to Dr. Yasumi Katayama, yasumi@ dokkyomed.ac.jp 


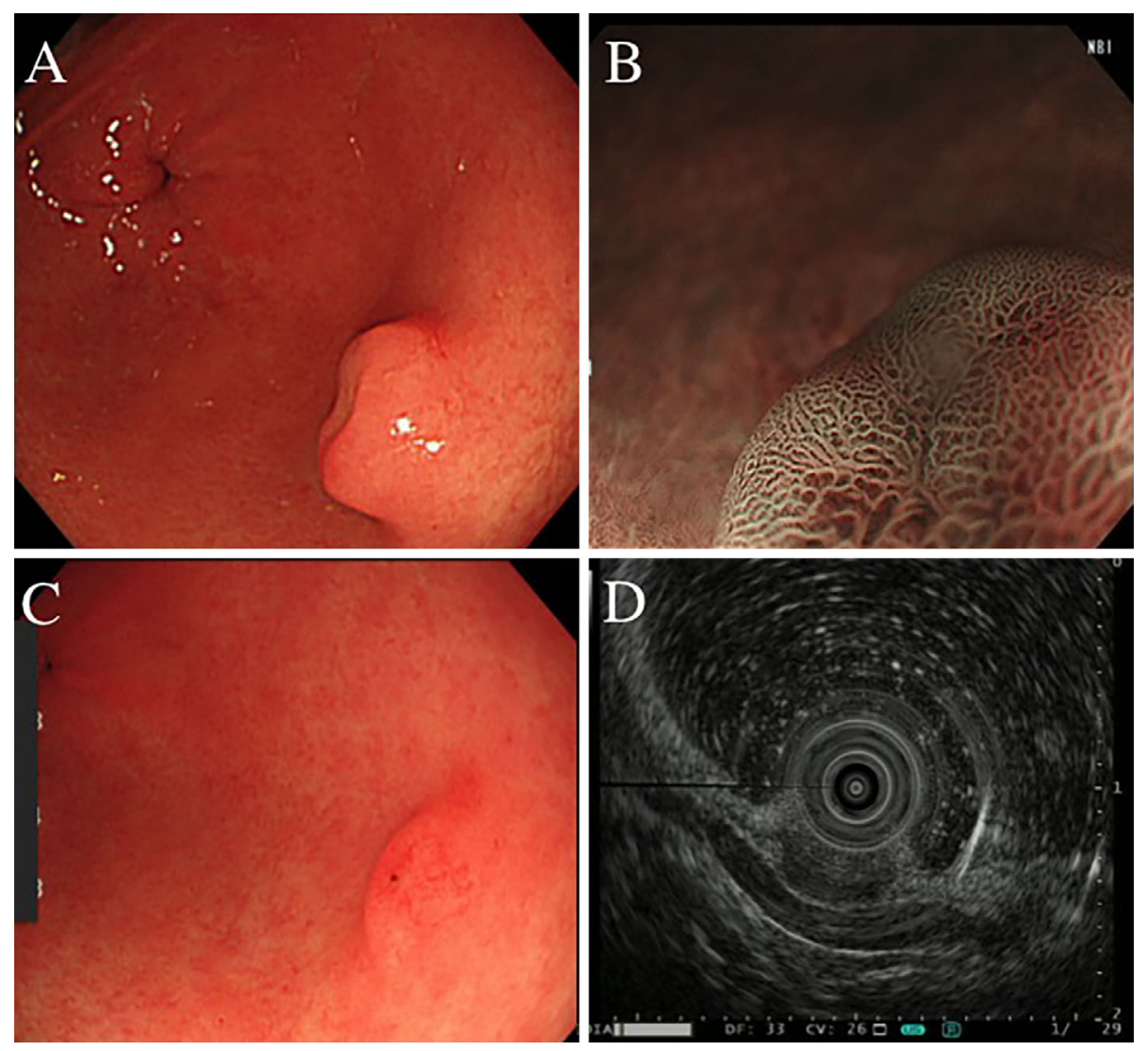

Figure 1. Esophagogastroduodenoscopy (EGD) findings. A: The most recent EGD findings show an approximately 10-mm submucosal tumor-like elevation in the gastric antrum that has obviously increased in size over time. B: Magnified image-enhanced endoscopic findings reveal no obvious mucosal irregularities. C: Small, elevated lesion in the gastric antrum determined by EGD four years earlier. D: Endoscopic ultrasound shows a continuous low-echoic mass in the second layer but no disruption of the third layer.

cosal involvement around the apical ulcerative area (Fig. 2B). Histological findings revealed the relatively paucicellular, uniform proliferation of spindle cells with oval-tospindle bland nuclei in fibromyxoid stroma-rich small vessels with muscularis mucosa involvement (Fig. 2C). Alcian blue stain highlighted the myxoid features of the stroma (Fig. 2D). The tumor cells were immunohistochemically diffusely positive for $\alpha$-smooth muscle actin (Fig. 3A), partially positive for muscle specific actin (HHF35) and heavy caldesmon, focally and weakly positive for CD10, and negative for desmin, CD34 (Fig. 3B), CD117, S-100 protein, HMB45 and pancytokeratin (AE1AE3). The tumor was resected en bloc by ESD with negative lateral and deep resection margins (Fig. 2B).

\section{Discussion}

Plexiform fibromyxomas are very rare mesenchymal tumors that are known by various names. Takahashi (1) described two hitherto unknown gastric mesenchymal tumors in 2007 and named them plexiform angiomyxoid myofibroblastic tumors (PAMTs). Two years later, Miettinen (2) described 12 fibromyxoid tumors in the gastric antrum that he named plexiform fibromyxoma. The World Health Organization classification included plexiform fibromyxoma in 2010 . Regardless of the controversial name $(4,5)$, only 30 to 60 plexiform fibromyxomas have been described to date $(3,6,7)$. The prevalence of these tumors has not been found to differ in terms of the sex or age of patients, and the tumors occur mainly in the gastric antrum $(2,3,6)$, but a few have been identified in the esophagus, duodenum, small intestine and gallbladder (8-11).

The mean size of these reported tumors (3) was $52 \mathrm{~mm}$, and they were treated at a far more advanced stage than that in our patient. Although such tumors cause symptoms such as abdominal pain, nausea, anemia and hematemesis, they are thought to remain asymptomatic unless they become large enough to form ulcers on the mucosal surface.

Histologically, such tumors exhibit plexiform growth of small spindle cells in myxoid or fibromyxoid stroma and are immunohistochemically KIT (-), DOG1 (-), CD34 (-), S100 $(-)$ and desmin (-), which helps to differentiate them from GIST, leiomyoma and schwannoma (2). Plexiform fibromyxoma is basically considered benign, but evidence has yet to confirm that this type of tumor remains benign, so long-term follow-up is required. The histological and im- 


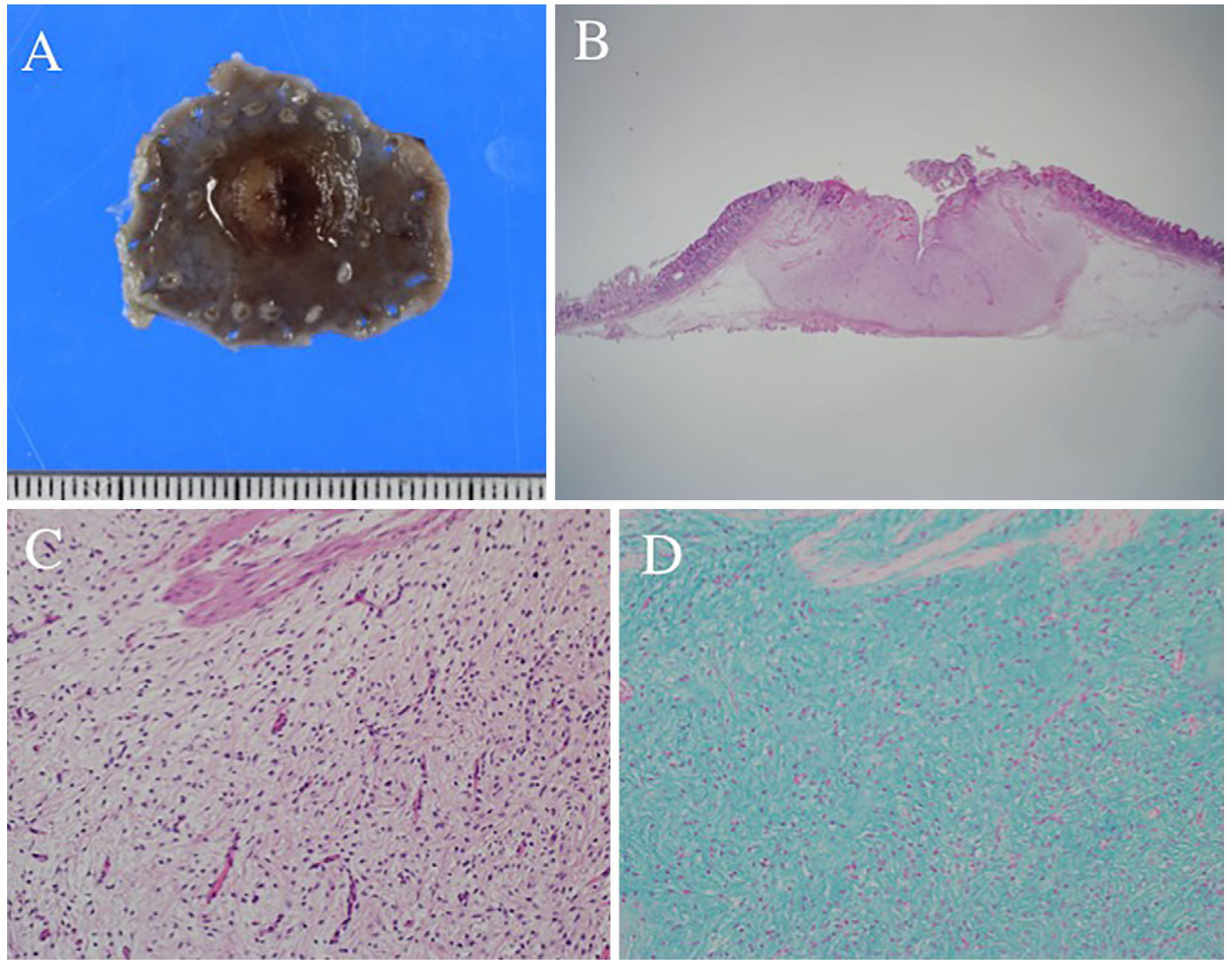

Figure 2. Pathological findings of resected tumor. A: Dome-shaped polypoid lesion measuring $11 \times 11 \mathrm{~mm}$ with apical ulcerative depression. B: Whole-mount view: Well-circumscribed, uninodular tumor with fibromyxomatous cut surface. C: High-power histologic view (400x): Relatively paucicellular, uniform proliferation of spindle cells with oval-to-spindle bland nuclei in fibromyxoid stromarich small vessels. D: Alcian blue stain reveals the myxoid features of stroma.
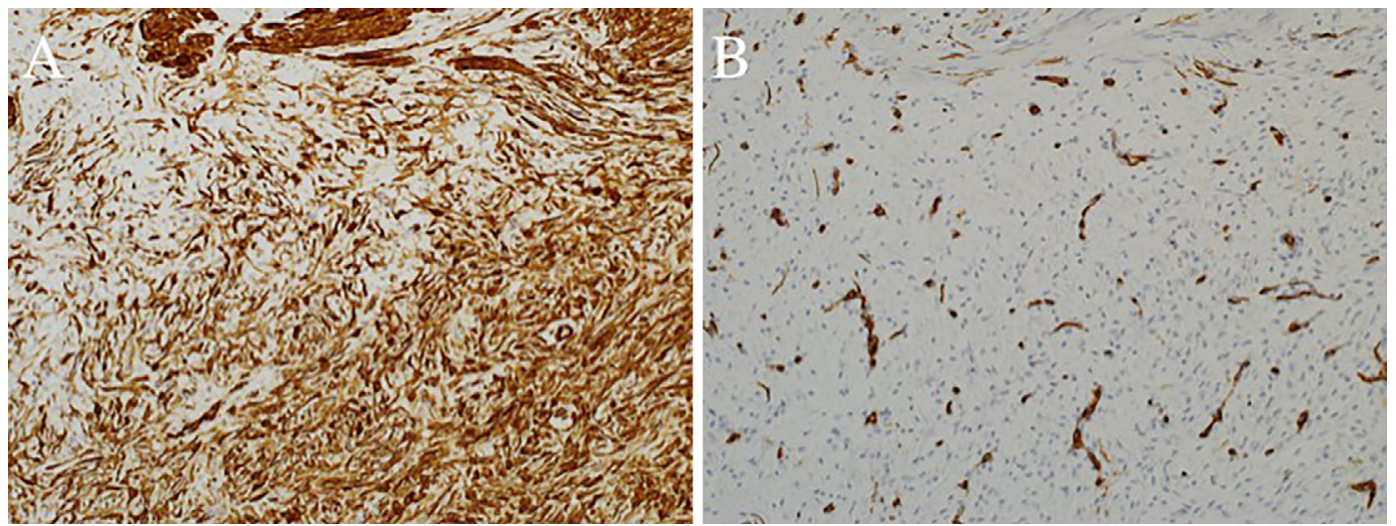

Figure 3. Immunohistochemical findings of tumor cells. $(400 \times)$. A: Tumor cells are diffusely positive for $\alpha$-smooth muscle actin. B: Tumor cells are negative for CD34, with positive staining of small vessel endothelials.

munochemical findings of the fibromyxomatous tumor in our patient revealed myofibroblastic cells with no evidence of malignancy. Although the high-power histologic features were consistent with reported gastric PAMT or plexiform fibromyxoma, the present tumor did not have the multinodular plexiform growth that is characteristic of such tumors. No uninodular disease concept has yet been proposed. Thus, we tentatively referred to the present tumor as a gastric uninodular fibromyxomatous tumor, stressing its singular nodularity and the fact that its size increased over time. This is the first report of uninodular fibromyxoma. This single nodule was treatable even at $11 \mathrm{~mm}$, but it might have had the potential to become multinodular should it have been allowed to grow larger. 
Because plexiform fibromyxomas often extend into the muscular layer, endoscopic treatment is not indicated, making laparotomy or laparoscopic surgery the preferred options $(3,6,7)$. Two endoscopic treatments for plexiform fibromyxoma have been attempted (12) as far as we can ascertain. However, preoperative EUS revealed a thickened muscle layer in one case, and the resection margin was unknown, so the indication for endoscopic treatment is unclear. Tumor disruption of the third layer was not detected on EUS in our patient. The tumor was mainly located in the second layer and resected by ESD. The vertical stump was pathologically negative. No recurrence was observed on follow-up endoscopy or CT after six months.

In conclusion, we herein reported a case of uninodular gastric fibromyxoma in which the size increased over time.

\section{The authors state that they have no Conflict of Interest (COI).}

\section{References}

1. Takahashi Y, Shimizu S, Ishida T, et al. Plexiform angiomyxoid myofibroblastic tumor of the stomach. Am J Surg Pathol 31: 724728, 2007.

2. Miettinen M, Makhlouf HR, Sobin LH, Lasota J. Plexiform fibromyxoma: a distinctive benign gastric antral neoplasm not to be confused with a myxoid GIST. Am J Surg Pathol 33: 1624-1632, 2009.

3. Kane JR, Lewis N, Lin R, et al. Plexiform fibromyxoma with cotyledon-like serosal growth: a case report of a rare gastric tumor and review of the literature. Oncol Lett 11: 2189-2194, 2016.
4. Takahashi Y, Suzuki M, Fukusato T. Plexiform angiomyxoid myofibroblastic tumor of the stomach. World J Gastroenterol 16: 2835-2840, 2010.

5. Li P, Yang S, Wang C, Li Y, Geng M. Presence of smooth muscle cell differentiation in plexiform angiomyxoid myofibroblastic tumor of the stomach: a case report. Int J Clin Exp Pathol 7: 823827, 2014.

6. Quero G, Musarra T, Carrato A, et al. Unusual focal keratin expression in plexiform angiomyxoid myofibroblastic tumor: a case report and review of the literature. Medicine (Baltimore) 95: e 4207, 2016.

7. Szurian K, Till H, Amerstorfer E, et al. Rarity among benign gastric tumors: plexiform fibromyxoma - report of two cases. World J Gastroenterol 23: 5817-5822, 2017.

8. Daum O, Jirasek T, Grossmann P, Mukensnabl P, Michal M. Plexiform fibroma of the colon. Appl Immunohistochem Mol Morphol 18: 483-484, 2010.

9. Duckworth LV, Gonzalez RS, Martelli M, Liu C, Coffin CM, Reith JD. Plexiform fibromyxoma: report of two pediatric cases and review of the literature. Pediatr Dev Pathol 17: 21-27, 2014.

10. Fassan M, Salmaso R, Saraggi D, et al. Plexiform fibromyxoma of the gallbladder. Pathologica 107: 181-184, 2015.

11. Moris D, Spanou E, Sougioultzis S, et al. Duodenal plexiform fibromyxoma as a cause of obscure upper gastrointestinal bleeding: a case report. Medicine (Baltimore) 96: e5883, 2017.

12. Kawara F, Tanaka S, Yamasaki T, et al. Gastric plexiform fibromyxoma resected by endoscopic submucosal dissection after observation of chronological changes: a case report. World J Gastrointest Oncol 9: 263-267, 2017.

The Internal Medicine is an Open Access journal distributed under the Creative Commons Attribution-NonCommercial-NoDerivatives 4.0 International License. To view the details of this license, please visit (https://creativecommons.org/licenses/ by-nc-nd/4.0/).

(C) 2019 The Japanese Society of Internal Medicine Intern Med 58: 2015-2018, 2019 